\title{
ON THE VIETORIS-BEGLE THEOREM ${ }^{1}$
}

\author{
BY D. G. BOURGIN
}

Communicated by Raoul Bott, January 12, $1970^{2}$

The usual forms of the Vietoris-Begle theorem require vanishing of the first p-cohomology groups of the antecedents of points under the mapping. The present work utilizes a multi-cone construction to yield a variety of results when either one cohomology group alone vanishes or when several cohomology groups, not necessarily including that of dimension 0 , vanish. Some converse theorems are obtained relating the global conditions on the cohomology groups of the space and the vanishing of some of the cohomology groups of point antecedents. These results demand special conditions on the space and its map and trivial examples show that otherwise the theorems obtained are invalid. For the metric case Borsuk [1] used a somewhat different cone construction. His space and map restrictions are much stronger than those introduced here.

The cohomology groups are understood to be the reduced groups. The coefficient group is a fixed Abelian group. Throughout let $f: X \rightarrow Y$ be surjective and continuous and $X$ and $Y$ are compact Hausdorff. Denote by $X_{y}$ the set $\{x \mid f(x)=y\}$. Write $I_{y}$ for the segment [01] over $y$ and $\Pi_{Y_{0}}$ for the product $\prod_{y \in Y_{0}} I_{y}, Y_{0} \subset Y$ in the usual Tychonoff topology. If $Y_{0}=Y$ write $\Pi$. Thus the elements of $\Pi_{Y 0}$ are functions, $\psi$, on $Y_{0}$ to $I$. Designate by $1_{y_{0}}$ the function for which $1_{y_{0}}(y)=0, \quad y \neq y_{0}$ and $1_{y_{0}}\left(y_{0}\right)=1$ and let $J_{y_{0}}=\left\{\psi \mid \psi(y)=0, \quad y \neq y_{0}\right.$, $\left.\psi\left(y_{0}\right) \in I_{y}\right\}$. Denote by $*$ the identically 0 function. Let $P\left(Y_{0}\right)$ $=X \times \Pi\left(Y_{0}\right)$ with the product topology and write $B\left(y_{0}\right)=X_{y}=J_{v} \subset P$. The cylinder set $B$ is $\bigcup_{y \in Y_{0}} B_{y} \subset P$. Intuitively $B$ consists of cylinders sticking out in different directions.

It is easy to show that $B$ is closed in the compact space $P$ and so is compact. It is evidently Hausdorff also. Now collapse the roofs of the cylinders, $B_{y}$, in $B$ to yield cones. More precisely identify $X_{y} \times 1_{y}$ to a peak point denoted by $x_{y}^{*}$. The resulting cone is denoted by $X_{v}^{*}$ and the collection of cones by ' $X$ where we assume the identification or quotient topology. Then

Lemma $1 . ' X$ is compact.

AMS Subject Classifications. Primary 5530, 5542.

Key Words and Phrases. Vietoris-Begle theorem, almost p-solid, finely sectioned, acyclic, Alexander cohomology, Tychonoff topology.

${ }^{1}$ Research supported by National Science Foundation Grant GP 9494.

${ }^{2}$ Original manuscript submitted on October 17, 1969. 
This is a consequence of the fact that ' $X$ is a (continuous) map of $B$ under the identification mapping. It is to be noted that the cluster points of cone vertices in ' $X$ lie in $X \times *$. Thus let $C=\left\{X_{v}^{*} \mid y \in Y_{1}\right\}$ be a nonfinite collection. Then if $(\bar{x}, *)$ is a cluster point of $\left\{\left(x_{y}, *\right) \mid y \in Y_{1}\right\}$, $(\bar{x}, *)$ is a cluster point of $C$.

However ' $X$ need not be Hausdorff. For example suppose $X$ is the unit planar disk and $Y$ is the segment [01]. If for some $y, x$ and $x^{\prime}$ are distinct points of $X_{y}$ they cannot be separated since their neighborhoods contain a nondenumerable number of common peak points.

We therefore introduce a modified ' $X$. Thus define

$$
X^{*}\left(Y_{0}\right)=\bigcup_{y \in Y_{0}} X_{y}^{*} \cup \underset{y \in Y_{0}}{\bigcup} X_{y}
$$

with the topology induced by that of ' $X$. Evidently $X^{*}\left(Y_{0}\right)$ is compact.

Interpret "almost all" in the sense of: up to a finite subset. Choose $Y_{0}$ so that for arbitrary $x_{0} \in X$ and for every neighborhood $N\left(x_{0}\right)$, there is a neighborhood $N^{\prime}\left(x_{0}\right)$ such that for almost all $y$ in $\left\{y \mid X_{y} \cap N^{\prime}\left(x_{0}\right) \neq \varnothing\right\} \cap Y_{0}, X_{y} \subset N\left(x_{0}\right)$.

Lemma 2. $X^{*}\left(Y_{0}\right)$ is Hausdorff.

We say that $X, f$ is almost $p$-solid if $Y_{0}$ defined above can be taken as $\left\{y \mid X_{y}\right.$ is not $r$-acyclic for some $\left.r<p\right\}$. Denote this $Y_{0}$ by $Y_{p}$. If $Y$ satisfies the condition for $Y_{0}$ then $(X, f)$ is finely sectioned.

TheOREM 3. Let $X, f$ be almost p-solid and suppose $H^{p}\left(X_{y}, G\right)=0$. Then $H^{m}(Y, G) \stackrel{f^{*}}{\rightarrow} H^{m}(X, G)$ is an epimorphism for $m=p$ and a monomorphism for $m=p+1$.

Corollary 4. If the condition $H^{p+1}\left(X_{y}, G\right)=0$ is added to the hypotheses in Theorem 3 then $f^{*}$ is an epimorphism for $m=p$, an isomorphism for $m=p+1$ and a monomorphism for $m=p+2$.

Corollary 5. If $X, f$ is finely sectioned and $H^{m}\left(X_{y}, G\right)=0$ for $m=p, p+1$ then $H^{p}(Y, G) \approx H^{p}(X, G)$.

This last corollary is known to Deleanu [3] (the idea of whose proof is somewhat similar to that of our Theorem 3) and generalizes Borsuk [1].

The proof of Theorem 3 is sketched: Write $X^{*}$ for $X^{*}\left(Y_{p}\right)$. Since $X_{y}^{*}, y \in Y_{p}$, is a cone it is acyclic. Hence the exact cohomology sequence for the pair $X_{y}^{*}, X_{y}$ yields

$$
H^{m}\left(X_{y}, G\right) \approx H^{m+1}\left(X_{y}^{*}, X_{y}, G\right)
$$


for all $m$. Extend $f$ on $X$ to $Y$ to $F$ on $X^{*}$ to $Y$ by $F\left(X_{y}^{*}\right)=y \in Y_{p}$. Again since $X_{\nu}^{*}, y \in Y_{p}$, is acyclic and $X_{y}$ is acyclic through dimension $p$, the usual Vietoris-Begle Theorem [2] yields

$$
H^{m}\left(X^{*}, G\right) \stackrel{F^{*}(m)}{\approx} H^{m}(Y, G), \quad m \leqq p,
$$

and $F^{*}(p+1)$ is a monomorphism.

Let $\rho$ be a finite subset of $Y$ and define

$$
X_{p}^{*}=\bigcup_{y \in \rho \cap Y_{p}}\left(X_{y} \cup\left(X^{*}-\bigcup_{y \in \rho \cap Y_{p}} X_{y}^{*}\right)\right)
$$

which is easily seen to be compact and Hausdorff. Since Alexander cohomology with compact supports is equivalent to Cech cohomology the continuity theorem applies to the inverse system $\left\{X_{\rho}^{*}, p_{\rho}^{\rho^{\prime}}\right\}$ where the matching maps $p_{\rho}^{\rho^{\prime}}$ are inclusions and the ordering is by inverse inclusion. Furthermore, [4, p. 146] for Alexander cohomology with compact support $H(X, A, G) \approx H(X-A, G)$ with $A$ closed in the compact set $X$. Combining these facts with

$$
X^{*}, X=\underset{\sim}{\rightarrow}\left\{X^{*}, X_{\rho}^{*}, p_{\rho}^{p^{\prime}}\right\}
$$

yields eventually

Consider the diagram

$$
H^{m}\left(X^{*}, X, G\right)=\underset{\rightarrow}{\longrightarrow} \underset{y \in \rho \cap Y_{p}}{\oplus} H^{m-1}\left(X_{y}, G\right) .
$$

$$
\begin{aligned}
& H^{m}\left(X^{*}, X, G\right) \rightarrow H^{m}\left(X^{*}, G\right) \stackrel{\psi(m)}{\longrightarrow} H^{m}(X, G) \stackrel{\psi}{\rightarrow} H^{m+1}\left(X^{*}, X, G\right) \\
& F^{*}(m) \searrow_{H^{m}(Y, G)} \nearrow^{f^{*}(m)}
\end{aligned}
$$

where the row is exact and the triangle is commutative. From the acyclicity of $X_{y}^{*}, y \in Y_{p}$, follows that $F^{*}(p+1)$ is a monomorphism. From (5b) follows that $H^{p+1}\left(X^{*}, X, G\right)=0$. Hence $\psi(p+1)$ is a monomorphism. Since $f^{*}(p+1)=\psi(p+1) F^{*}(p+1)$ there results $f^{*}(p+1)$ is a monomorphism. The reasoning establishing $f^{*}(p)$ is an epimorphism is similar (but perhaps simpler since $F^{*}(p)$ is an isomorphism).

Corollary 6. If $(X, f)$ is almost p-solid and if $H^{m}\left(X_{y}, G\right)=0$, $m=p, \cdots, q$, then $f^{*}(m)$ is a monomorphism for $m=q+1$, an isomorphism for $m=p+1, \cdots, q$ and an epimorphism for $m=p$.

A converse theorem can be stated.

THEOREM 7. If $(X, f)$ is almost $p+1$-solid and $f^{*}(p)$ is an epimorphism while $f^{*}(p+1)$ is a monomorphism then $H^{p}\left(X_{y}, G\right)=0$. 
The commutativity of the triangle in $(5 c)$ implies, in view of the hypotheses, that $\psi(p)$ and $\psi(p+1)$ are respectively an epimorphism and a monomorphism. It can then be shown that this and (5b) guarantees the conclusion.

Corollary 8. If $(X, f)$ is almost $q+1$-solid and if $H^{m}(Y, G)$ $\stackrel{f^{*}(m)}{\rightarrow} H^{m}(X, G)$ where $f^{*}(m)$ is an epimorphism for $m=p$, an isomorphism for $p<m \leqq q$ and a monomorphism for $m=q+1$ then $H^{m}\left(X_{y}, G\right)$ $=0, m=p, \cdots, q$.

That the restriction on $(X, f)$ cannot be summarily dropped is evident from the example cited earlier where $H^{1}(X) \approx H^{1}(Y)=0$, yet $H^{1}\left(X_{y}\right) \neq 0, y \neq 0$.

The next theorems extend some recent results of BialynickiBirula [5].

Suppose $X \stackrel{f}{\rightarrow} Y \stackrel{0}{\rightarrow} Z$ where $X$ and $Y$ are compact while $Z$ is arbitrary and $f$ and $g$ are surjective with $f$ continuous while $g$ need not be though $g^{-1}(z)$ is compact. Write $h=g f$.

THEOREM 9. If $H^{m}\left(h^{-1} z, G\right) \approx H^{m}\left(g^{-1} z, G\right), m=p, \cdots, q+1$, and if $(X, f)$ is almost $q+1$-solid then $f^{*}(m)$ is an epimorphism for $m=p$, an isomorphism for $p<m \leqq q$ and a monomorphism for $m=q+1$.

Since $h^{-1} z$ maps by $f$ onto $g^{-1} z$, the hypotheses when applied to these spaces are those of Corollary 7 where

$$
H^{m}\left(f^{-1}(y), G\right)=0, \quad m=p, \cdots, q,
$$

and Corollary 6 then applies.

Again a converse theorem is valid.

TheOREM 10. If $X, f$ is almost $q+1$-solid and $f^{*}(m): H^{m}(Y, G)$ $\rightarrow H^{m}(X, G)$ is an epimorphism for $m=p$, an isomorphism for $p<m \leqq q$ and a monomorphism for $m=q+1$ then

$$
k^{*}(m): H^{m}\left(g^{-1}(z), G\right) \rightarrow H^{m}\left(h^{-1}(z), G\right)
$$

is an epimorphism, an isomorphism and a monomorphism for the same values as $f^{*}(m)$.

There are applications to relations. For instance suppose again that $X$ and $Y$ are compact and that $\Gamma \subset Y \times Y$ is a closed subset. Denote by $\pi$ the projection onto the first coordinate, i.e. $\pi\left(y_{1}, y_{2}\right)=y_{1}$. Let $L\left(y_{2}\right)=\left\{\left(y_{1}, y_{2}\right) \mid\left(y_{1}, y_{2}\right) \in \Gamma\right\}$ and write $L\left(Y_{2}\right)=U_{y_{2} \in Y_{2}} L\left(y_{2}\right)$.

Theorem 11. Let $f: X \rightarrow Y$ be surjective and let $(X, f)$ be finely sectioned. Suppose $f^{*}(m): H^{m}(L(y)) \rightarrow H^{m}\left(f^{-1} \pi L(y)\right)$ is an epimorphism for $m=p$, an isomorphism for $p<m \leqq q$ and a monomorphism for $m$ 
$=q+1$. Let $\pi \Gamma=Y^{1}$. Then $k^{*}(m): H^{m}\left(Y^{1}\right) \rightarrow H^{m}\left(f^{-1} Y^{1}\right)$ is an epimorphism, an isomorphism and a monomorphism for the same $m$ values as $f^{*}(m)$.

The definition of almost $p$-solid can be given an alternative form. Let $\Delta$ be the diagonal of $X \times X$ and let $V(\Delta)$ be a vicinity. We say $X, f$ satisfies $\Pi$ for $Y_{0}$, if for any $V(\Delta)$ almost all sets $\left\{X_{v} \times X_{y} \mid y \in Y_{0}\right\}$ lie in $V(\Delta)$.

LEMMA 12. (a) $X, f$ is almost $p$-solid iff $X, f$ satisfies $\Pi$ for $Y_{0}$.

(b) If $X, f$ is almost p-solid then for arbitrary open $N\left(x_{0}\right)$ the open set $N^{\prime}\left(X_{0}\right)$ in the defining condition for almost p-solidity need satisfy $N^{\prime}\left(x_{0}\right) \subset N\left(x_{0}\right)$ only.

For the case of $X$, a compactum, it is easy to show that if $X, f$ is almost $p$-solid then $Y_{0}$ is denumerable and hence 0 -dimensional and therefore results such as those of [1] and Theorems 3 and 6 are included in those of [6]. However this is no longer true for $X$ compact but nonmetric. An example is $X=S^{n} \bigvee X^{0}, n>2$, where $X^{0}$ is $S^{1} \times I$ under a certain partial ordering with the relative topology of $X^{0}$ being given by the open ordered interval topology and $S^{n}$ is attached to $I$ by one point at $0 \in I$. With $Y=S^{n} \bigvee I$ and $f$ the identity on $S^{n}$ and a projection on $X^{0}$ to $Y$ it can be shown that our Theorem 3 for instance yields an epimorphism for $m=2$ and an isomorphism for $m=3$ while [6] does not cover $m=2$ and merely asserts an epimorphism for $m=3$.

Theorem 3 and Corollaries 4 and 5, for instance, are valid for weakened conditions. Specifically $X$ and $Y$ need only be locally compact when cohomology with compact supports is used. Again $X$ and $Y$ may be paracompact provided $f$ is a closed mapping satisfying $f^{-1}\left(Y_{p}\right)$ is compact and supports are the closed sets.

\section{REFERENCES}

1. K. Borsuk, Concerning some upper-continuous decompositions of compacta, Bull. Acad. Polon. Sci. Sér. Sci. Math. Astronom. Phys. 11 (1963), 499-503. MR 30 \#3470.

2. G. E. Bredon, Sheaf theory, McGraw-Hill, New York, 1967. MR 36 \#552.

3. A. Deleanu, Unpublished.

4. D. G. Bourgin, Modern algebraic topology, MacMillan, New York, 1963. MR 28 \#3415.

5. A. Bialynicki-Birula, On Vietoris mapping theorem and its inverse, Fund. Math. 53 (1963/64), 135-145. MR $29 \# 606$.

6. E. G. Skljarenko, Some applications of the theory of sheaves in general topology, Uspehi Mat. Nauk 19 (1964), no. 6 (120), 47-70= Russian Math. Surveys 19 (1964), no. 6, 41-62. MR $30 \# 1490$.

University of Houston, Houston, Texas 77004 